\title{
THE ATIYAH-JONES CONJECTURE
}

\author{
C. P. BOYER, J. C. HURTUBISE, B. M. MANN, AND R. J. MILGRAM
}

\begin{abstract}
The purpose of this note is to announce our proof of the AtiyahJones conjecture concerning the topology of the moduli spaces of based $S U(2)-$ instantons over $S^{4}$. Full details and proofs appear in our paper [BHMM1].
\end{abstract}

\section{INTRODUCTION}

In 1978 Atiyah and Jones [AJ] proved a foundational theorem on the lowdimensional homology of the moduli space of based $S U(2)$ instantons on $S^{4}$ and made a number of conjectures. In this note we briefly explain how, using methods of algebraic geometry and algebraic topology, we analyze the global geometry of these moduli spaces, which we denote by $\mathscr{M}_{k}$, and prove the AtiyahJones conjectures. More precisely, let $P_{k}$ be a principal $S U(2)$ bundle over $S^{4}$ with second Chern class $k$. Let $\mathscr{M}_{k}$ be the space of based gauge equivalence classes of connections on $P_{k}$ that satisfy the Yang-Mills self-duality equations of $S U(2)$ gauge theory. There is a natural forgetful map $\vartheta_{k}: \mathscr{M}_{k} \rightarrow \mathscr{B}_{k}$ where $\mathscr{B}_{k}$ is the space of based equivalence classes of all connections in $P_{k}$. Atiyah and Jones [AJ] showed that $\mathscr{B}_{k}$ is homotopy equivalent to $\Omega_{k}^{3} S U(2)$ and proved

Theorem [Atiyah-Jones]. $\vartheta_{k}$ induces a homology surjection

$$
H_{t}\left(\mathscr{M}_{k}\right) \stackrel{\left(\vartheta_{k}\right)_{t}}{\rightarrow} H_{t}\left(\Omega_{k}^{3} S U(2)\right) \rightarrow 0
$$

for $t \leq q=q(k) \ll k$.

The main point of the Atiyah-Jones result is that the homology of $\Omega^{3} S U(2)$ is completely known so that the surjection sheds some light, for small values of $t$ relative to $k$, on the heretofore unknown groups $H_{t}\left(\mathscr{M}_{k}\right)$. Notice that, as $k$ increases, the target spaces $\Omega_{k}^{3} S U(2) \cong \Omega_{k+1}^{3} S U(2)$ remain homotopy equivalent, whereas the topology of the $\mathscr{M}_{k}$, which are $4 k$ dimensional complex manifolds, changes as $k$ varies. Atiyah and Jones then made the following conjectures:

1. $\left(\vartheta_{k}\right)_{t}$ is a homology isomorphism for $t \leq q(k)$.

2. The range of the surjection (isomorphism) $q=q(k)$ can be explicitly determined as a function of $k$.

3. The homology statements can be replaced by homotopy statements in both conjectures 1 and 2 .

The last and strongest statement became commonly known as the AtiyahJones conjecture. Notice that, while it is a simple fact that there are natural

Received by the editors August 14, 1991.

1991 Mathematics Subject Classification. Primary 32G13, 55P35; Secondary 14F05, 81E10.

During the preparation of this work the first and third authors were supported by NSF grants, the second author by an NSERC grant, and the fourth author by MSRI and an NSF grant. 
maps $j_{k}: \Omega_{k}^{3} S U(2) \stackrel{\simeq}{\rightarrow} \Omega_{k+1}^{3} S U(2)$, there is no obvious map on the $\mathscr{M}_{k}$ level that raises the instanton number $k$. Rather, it follows from analytic results of Taubes [T1] that there are compatible inclusions $l_{k}: \mathscr{M}_{k} \rightarrow \mathscr{M}_{k+1}$ on the instanton moduli space level such that the following diagram homotopy commutes

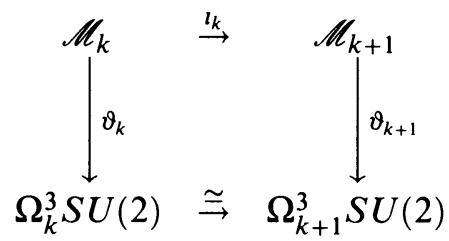

This diagram permits one to take direct limits and hence formulate the stable version of the Atiyah-Jones conjecture. This was proven by Taubes [T2].

Theorem [Taubes Stability Theorem]. Let $\mathscr{M}_{\infty}$ be the direct limit of the $\mathscr{M}_{k}$ 's under the inclusions $l_{k}$ and let $\vartheta: \mathscr{M}_{\infty} \rightarrow \Omega_{0}^{3} S U(2)$ be the direct limit of the maps $\vartheta_{k}$ in diagram (1.1). Then $\vartheta$ is a homotopy equivalence.

Using different methods Gravesen [Gra] independently proved that $\vartheta$ is a homology equivalence. However, these are stable results and do not directly address the structure of $H_{t}\left(\mathscr{M}_{k}\right)$ or $\pi_{t}\left(\mathscr{M}_{k}\right)$ for any fixed $t$ or $k$. In another direction, [BoMa] showed that $\left(\vartheta_{k}\right)_{t}$ is a surjection in $\mathbb{Z} / p$ homology for $t \leq k$ but the techniques used there do not extend to analyze the kernel.

In [BHMM1] we prove the following theorem, which settles conjectures 1,2 , and 3 of Atiyah and Jones in the affirmative.

Theorem A. For all $k>0$, the natural inclusion $\vartheta_{k}: \mathscr{M}_{k} \rightarrow \Omega_{k}^{3} S U(2)$ is a homotopy equivalence through dimension $q=q(k)=[k / 2]-2$.

By a homotopy equivalence through dimension $q(k)$ we mean that $\vartheta_{k}$ induces an isomorphism $\pi_{t}\left(\mathscr{M}_{k}\right) \cong \pi_{t}\left(\Omega_{k}^{3} S U(2)\right)=\pi_{t+3}(S U(2))$ for $t \leq q(k)$. Thus, for example, the low-dimensional homotopy groups of $\mathscr{M}_{k}$ are finite. The statement in homotopy implies the weaker statement that $\vartheta_{k}$ induces an isomorphism $H_{t}\left(\mathscr{M}_{k} ; A\right) \cong H_{t}\left(\Omega_{k}^{3} S U(2) ; A\right)$ for $t \leq q(k)$ and any coefficient group $A$. Here, the homology groups on the right are completely known.

Actually, Theorem A is a corollary of the main topological result in [BHMM1].

Theorem B. For all $k>0$, the inclusion $l_{k}: \mathscr{M}_{k} \rightarrow \mathscr{M}_{k+1}$ is a homotopy equivalence through dimension $q=q(k)=[k / 2]-2$.

These topological results are actually formal consequences of our detailed geometric analysis of $\mathscr{M}_{k}$. This is explained in the following section. Lastly, it is likely that the explicit bound $q(k)$ can be improved as explained at the end of the next section.

\section{OUTLINE OF PROOFS}

A key tool in our approach is a theorem of Donaldson [D], that allows us to work in a purely holomorphic context and study a certain moduli space of holomorphic bundles. To prove Theorems A and B we study this equivalent holomorphic moduli space by following the program used in [MM1] and [MM2] to study the topology of holomorphic maps from the Riemann sphere into flag manifolds. This should not be surprising since these holomorphic mapping 
spaces correspond, by results of [Hu2] and [HM], to moduli spaces for $S U(n)$ monopoles. The main idea is to construct a stratification of the entire moduli space by smooth manifolds, each with an orientable normal bundle, and each contained in the closure of higher dimensional strata. These strata are then organized into a filtration, and the resulting spectral sequence is analyzed to prove homology versions of Theorems A and B. More precisely, we prove

Theorem C. For each $k$ and all coefficient rings $A$, there are homology Leray spectral sequences $E^{r}\left(\mathscr{M}_{k} ; A\right)$ converging to filtrations of $H_{*}\left(\mathscr{M}_{k} ; A\right)$ with identifiable $E^{1}$ terms (see [BHMM1] for the precise statement). Furthermore, the inclusion $\imath_{k}: \mathscr{M}_{k} \rightarrow \mathscr{M}_{k+1}$ induces a map of spectral sequences $\imath_{k}(r): E^{r}\left(\mathscr{M}_{k} ; A\right) \rightarrow$ $E^{r}\left(\mathscr{M}_{k+1} ; A\right)$. In particular, differentials in these spectral sequences are natural with respect to $l_{k}(r)$.

Next, we show that the map $l_{k}$ induces an isomorphism of the $E^{2}$ terms in Theorem $\mathrm{C}$ through dimension $q(k)+2$. Since differentials are natural, it follows that $l_{k}$ induces a homology equivalence through dimension $q(k)+1$. This gives the homology version of Theorem B, namely,

Theorem D. For all $k>0$ and all coefficients $A$, the inclusion $l_{k}: \mathscr{M}_{k} \rightarrow \mathscr{M}_{k+1}$ induces an isomorphism in homology $\left(l_{k}\right)_{t}: H_{t}\left(\mathscr{M}_{k} ; A\right) \cong H_{t}\left(\mathscr{M}_{k+1} ; A\right)$ for $t \leq q=q(k)+1$.

Combined with the homology Taubes-Gravesen stability theorem, Theorem D implies the homology version of Theorem A. However, as $\mathscr{M}_{k}$ and $\Omega^{3} S^{3}$ are not simply connected, Theorems $A$ and $B$ do not trivially follow from their homology analogs. Thus, we analyze the induced map on the universal covers $\left(\tilde{\vartheta}_{k}\right)_{t}: H_{*}\left(\widetilde{\mathscr{M}_{k}} ; \mathbb{Z}\right) \rightarrow H_{t}\left(\widetilde{\Omega}^{3} S^{3} ; \mathbb{Z}\right)$ and show this is an isomorphism for $t \leq$ $q(k)$. This now implies Theorems A and B.

Thus, to complete the outline of the proof we need to explain how we prove Theorem C. It is nontrivial to obtain a stratification for $\mathscr{M}_{k}$ that is suited to this type of topological analysis. In fact, much of the work in [BHMM1] is devoted to understanding the geometry of $\mathscr{M}_{k}$ in sufficient detail so that we can construct such a stratification. As mentioned above the starting point for this analysis is a result of Donaldson [D] saying that the framed $S U(2)$ instanton moduli space is equivalent to the moduli space of rank two holomorphic vector bundles on $\mathbb{P}^{2}$ with $c_{1}=0$ that are trivial when restricted to a fixed line $\ell$ with a fixed trivialization there. Moreover, as was noticed in [A] and [Hu1], it is convenient to perform a birational transformation on the line $\ell$ to obtain a surface ruled by lines, for example, $\mathbb{P}^{1} \times \mathbb{P}^{1}$. Let $\mathscr{M}_{k}(M, S)$ denote the moduli space of isomorphism classes of rank 2 holomorphic bundles on $M$ with $c_{1}=0$ and $c_{2}=k$ that have a fixed trivialization on $S$. Then there are diffeomorphisms

$$
\mathscr{M}_{k} \simeq \mathscr{M}_{k}\left(\mathbb{P}^{2}, \ell\right) \simeq \mathscr{M}_{k}\left(\mathbb{P}^{1} \times \mathbb{P}^{1}, \ell_{1} \vee \ell_{2}\right) \text {. }
$$

Thus, the geometry of $\mathscr{M}_{k}$ is described by isomorphism classes of certain framed semistable holomorphic rank 2 vector bundles $E$ over $\mathbb{P}^{1} \times \mathbb{P}^{1}$. It is known that such $E$ are trivial on almost all lines $\mathbb{P}^{1} \times\{x\}$ and that the structure of $E$ is determined by its behaviour on neighbourhoods of a finite number of jumping lines $\mathbb{P}^{1} \times\left\{x_{i}\right\}$, lines over which the holomorphic structure of $\mathrm{E}$ is that of a sum of line bundles $\mathscr{O}(h) \oplus \mathscr{O}(-h)$ with $h>0$. In particular, if $\{\infty\}$ in the second $\mathbb{P}^{1}$ corresponds to the line $\ell_{1}$, then the possible jumping 
lines are parameterized by the complex plane $\mathbb{C} \simeq \mathbb{P}^{1}-\{\infty\}$. At each jumping line there is an associated integer $m$ called the multiplicity. Let $Q_{m}$ denote the framed isomorphism classes (on a neighbourhood of a jumping line) of framed jumps of multiplicity $m$. To each instanton $\omega$ is thus associated an isomorphism class $\left[E_{\omega}\right]$ of bundles $E_{\omega}$ and, in turn, to each [ $\left.E_{\omega}\right]$ is associated a point in a labelled configuration space. Elements of this space are given by configurations of points $z_{i}$ in the complex plane $\mathbb{C}$ determined by a finite number of nontrivial jumping lines each labelled by elements $l_{i} \in Q_{m_{i}}$. Here the total charge $k$ is the sum of the multiplicities. Thus, as shown in [Hul], there is a natural holomorphic projection $\Pi: \mathscr{M}_{k} \rightarrow S P^{k}(\mathbb{C})$ that associates to any equivalence class of framed bundles $[E]$ its divisor of jumping lines $\sum_{i=1}^{r} m_{i} z_{i}$ in $S P^{k}(\mathbb{C})$. The fibre at a point $z \in S P^{k}(\mathbb{C})$ with multiplicity $\left(m_{1}, \ldots, m_{r}\right)$ is the product $Q_{m_{1}} \times \cdots \times Q_{m_{r}}$ where $Q_{m}$ denotes the space of equivalence classes of framed jumps of multiplicity $m$. This is the "pole and principal part" picture of Segal-Gravesen [Gra].

However, as each $Q_{m}$ is not necessarily a smooth manifold, this decomposition of $\mathscr{M}_{k}$ is yet not sufficiently fine to apply the techniques of [MM1, MM2]. Therefore, a refined local analysis of $Q_{m}$ is required. To each multiplicity $m_{i}$ we associate finite sequences $G=\left(h_{0}, h_{1}, \ldots, h_{l-1}\right)$ of decreasing integers called "heights" such that $\sum_{j=0}^{l-1} h_{j}=m_{i}$. We refer to these sequences $G$ as "graphs" and show that to each framed jumping line of multiplicity $m$ we can associate a unique graph $G$. This is done by setting, for each $k$,

$$
h_{k}=\operatorname{dim}\left\{\begin{array}{c}
\text { sections of } E \otimes \mathscr{O}(-1) \text { on the jumping line which extend } \\
\text { to the } k \text { th formal neighbourhood of the line in } \mathbb{P}^{1} \times \mathbb{P}^{1}
\end{array}\right\} .
$$

We thus obtain a decomposition $Q_{m}=\bigsqcup_{G} F J_{G}$ where each $F J_{G}$ is the collection of framed jumps of a fixed graph type and the union is taken over all graphs $G$ of multiplicity $m$. Most importantly, we prove

Theorem E. Let $G$ be a graph with heights $h=h_{0} \geq h_{1} \geq \cdots \geq h_{l-1}>h_{l}=0$, length $l$, and multiplicity $m=\sum h_{i}$. The space of framed jumps $F J_{G}$ with graph $G$ is a smooth complex manifold of complex dimension $2 m+l$.

Let $S_{G_{1}, \ldots, G_{r}}$ denote the set of equivalence classes of framed holomorphic bundles with $r$ jumping lines $\left(L_{1}, \ldots, L_{r}\right)$ with graphs $\mathscr{G}(r)=\left(G_{1}, \ldots, G_{r}\right)$, respectively. Thus, we have the disjoint union decomposition $\mathscr{M}_{k}=\bigsqcup S_{\mathscr{G}}$. Furthermore, the map $\Pi$ now restricts to a map $\Pi_{\mathscr{G}(r)}: S_{\mathscr{G}(r)} \rightarrow \mathbb{D P}^{r}(\mathbb{C})$ with fibre $F J_{G_{1}} \times \cdots \times F J_{G_{r}}$. Here $\mathbb{D P}^{r}(\mathbb{C})$ is the deleted product; that is, the space of $r$ distinct unordered points in $\mathbb{C}$, which is a smooth complex manifold of complex dimension $r$. From Theorem E follows

Theorem F. $S_{\mathscr{G}(r)}$ is a smooth complex variety of complex dimension $2 k+l+r$.

We then prove that this decomposition of $\mathscr{M}_{k}$ by the $S_{\mathscr{G}}$ submanifolds is a stratification of the type required. In particular, it is necessary to know the codimension of each stratum (which is given in the last theorem), that there is a natural lexicographical order on the graph types, and, with respect to that order, what the intersection of the normal bundle of a fixed stratum is with the other strata. In addition to checking these facts we verify that our constructions are all compatible with the stabilization maps $l_{k}$ in diagram (1.1). After combining these results Theorem $\mathrm{C}$ follows as in [MM1, MM2]. 
Finally, we note that the stability argument given here does not require explicit calculation of differentials in the spectral sequence given in Theorem $\mathrm{C}$ but uses only information at the $E^{1}$ level and naturality. In a sequel [BHMM2] we will examine the structure of the $E^{1}$ term and of the differentials much more carefully. This will permit us to both sharpen the explicit value of $q(k)$ and, more importantly, obtain much more detailed information about $H_{*}\left(\mathscr{M}_{k}\right)$ above the range of stability.

\section{BIBLIOGRAPHY}

[A] M. F. Atiyah, Instantons in two and four dimensions, Comm. Math. Phys. 93 (1984), 437-451.

[AJ] M. F. Atiyah and J. D. Jones, Topological aspects of Yang-Mills theory, Comm. Math. Phys. 61 (1978), 97-118.

[BHMM1] C. P. Boyer, J. C. Hurtubise, B. M. Mann, and R. J. Milgram, The topology of instanton moduli spaces. I: The Atiyah-Jones conjecture, Ann. of Math. (2), (to appear).

[BHMM2] _ - The topology of instanton moduli spaces. II: The Toeplitz varieties, in preparation.

[BoMa] C. P. Boyer and B. M. Mann, Homology operations on instantons, J. Differential Geom. 28 (1988), 423-465.

[D] S. K. Donaldson, Instantons and geometric invariant theory, Comm. Math. Phys. 93 (1984), 453-461.

[Gra] J. Gravesen, On the topology of spaces of holomorphic maps, Acta Math. 162 (1989), 247-286.

[Hu1] J. C. Hurtubise, Instantons and jumping lines, Comm. Math. Phys. 105 (1986), 107-122.

[Hu2] _ The classification of monopoles for the classical groups, Comm. Math. Phys. 120 (1989), 613-641.

[HM] J. C. Hurtubise and M. K. Murray, On the construction of monopoles for the classical groups, Comm. Math. Phys. 122 (1989), 35-89.

[MM1] B. M. Mann and R. J. Milgram, Some spaces of holomorphic maps to complex Grassmann manifolds, J. Differential Geom. 33 (1991), 301-324.

[MM2] __ On the moduli space of SU(n) monopoles and holomorphic maps to flag manifolds, preprint, UNM and Stanford University, 1991.

[T1] C. H. Taubes, Path-connected Yang-Mills moduli spaces, J. Differential Geom. 19 (1984), 337-392.

[T2] _ The stable topology of self-dual moduli spaces, J. Differential Geom. 29 (1989), 163-230.

Department of Mathematics and Statistics, University of New Mexico, Albuquerque, New MeXico 87131

E-mail address: cboyer@gauss.unm.edu

Department of Mathematics and Statistics, McGill University, Montréal, Québec H3G 1M8, CANADA

E-mail address: hurtubis@gauss.math.mcgill.ca

Department of Mathematics and Statistics, University of New Mexico, Albuquerque, New MeXico 87131

E-mail address: mann@gauss.unm.edu

Department of Mathematics, Stanford University, Stanford, California 94305

E-mail address: milgram@gauss.stanford.edu 